\title{
EchoGéo
}

$10 \mid 2009$

La piraterie

\section{Une « Vision Mumbai » pour transformer la ville ou la difficulté à (re)penser la gouvernance métropolitaine}

Marie-Hélène Zérah

\section{CpenEdition}

Journals

Édition électronique

URL : https://journals.openedition.org/echogeo/11389

DOI : $10.4000 /$ echogeo.11389

ISSN : 1963-1197

Éditeur

Pôle de recherche pour l'organisation et la diffusion de l'information géographique (CNRS UMR 8586)

Référence électronique

Marie-Hélène Zérah, « Une « Vision Mumbai » pour transformer la ville ou la difficulté à (re)penser la gouvernance métropolitaine », EchoGéo [En ligne], 10 | 2009, mis en ligne le 15 septembre 2021 . consulté le 16 septembre 2021. URL : http://journals.openedition.org/echogeo/11389; DOI : https:// doi.org/10.4000/echogeo.11389

Ce document a été généré automatiquement le 16 septembre 2021.

EchoGéo est mis à disposition selon les termes de la licence Creative Commons Attribution - Pas d'Utilisation Commerciale - Pas de Modification 4.0 International (CC BY-NC-ND) 


\title{
Une « Vision Mumbai » pour transformer la ville ou la difficulté à (re)penser la gouvernance métropolitaine
}

\author{
Marie-Hélène Zérah
}

\section{Introduction}

1 Aujourd'hui, les villes sont des nœuds stratégiques dans le fonctionnement de l'économie mondiale (Sassen, 1994). Elles sont au cœur des restructurations des échelles de gouvernance (Brenner, 2004). Les acteurs de la sphère privée prennent une place croissante dans la production de la ville (Le Galès, 2005) et la poussée de la société civile s'accompagne d'une forte demande de démocratisation (Jouve, 2005). Dans ce contexte de multiplication des jeux d'acteurs, la question de la gouvernabilité et de la constitution de la ville comme acteur collectif s'impose dans les discussions sur l'avenir des villes. En France, le débat sur le Grand Paris illustre parfaitement ces enjeux. Deux positions s'opposent. La première considère que ces transformations sont une opportunité pour les villes de s'affirmer en tant qu'acteur politique (Bagnasco et Le Galès, 1997; Le Galès, 2003). D'autres auteurs sont plus circonspects. Ils pensent au contraire que certaines métropoles sont ingouvernables du fait d'une fragmentation accrue des intérêts et des espaces urbains (Jouve et Lefèvre, 2002). Ce débat souvent focalisé sur les villes nord-américaines et européennes se pose pour d'autres régions du monde, et en particulier pour l'Inde. En effet, les jeux d'échelle et les jeux d'acteurs s'y recomposent alors même que les villes s'étalent et se transforment économiquement. Tout d'abord, les villes prennent une place grandissante dans l'économie indienne : on estime qu'elles contribuent au PIB indien à la hauteur de $60 \%$. De plus, près de 286 millions de résidants y habitent, même si elles ne représentent que $30 \%$ de la population indienne totale. L'expansion des zones péri-urbaines (Dupont, 2007) transforme les grandes métropoles en de vastes ensembles urbains aux juridictions 
multiples. Enfin, la gouvernance urbaine est en profonde mutation avec, d'une part l'émergence des partenariats public-privé et de la société civile, d'autre part l'amendement constitutionnel sur la décentralisation politique de 1992 (Ruet et Tawa Lama-Rewal, 2009). Cette loi délègue plus de pouvoirs aux collectivités locales, mais celles-ci restent subordonnées aux gouvernements régionaux qui sont responsables des politiques urbaines. De plus, le gouvernement indien place les villes au cœur des stratégies de croissance et met en œuvre des programmes qu'il pilote directement à l'échelle fédérale (Kennedy et Zérah, 2008). Ainsi depuis décembre 2005, une mission nationale sur le renouvellement urbain, la Jawaharlal Nehru National Renewal Mission (JNNURM), a programmé des montants d'investissement exceptionnels pour mettre à niveau les infrastructures des grandes villes. Le déblocage de ces financements est conditionné à l'application par les gouvernements régionaux de réformes urbaines (dérégulation foncière, promotion des partenariats publics-privés). Ces tensions contradictoires entre centralisation et décentralisation posent donc de nouvelles questions sur la gouvernance de ces grands ensembles urbains et sur la manière dont est pensée la question de la métropolisation. L'ambition de cet article est, tout en traitant du cas spécifique de Mumbai, de donner des clés de compréhension de la montée de ces enjeux puis de s'attacher à montrer quels sont les problèmes de fonctionnement des institutions métropolitaines dans leur dimension "technique " d'aménagement, de planification et d'outils de production de la ville. On s'intéressera ensuite à la dimension politique avant de revenir sur la manière dont une «vision de Mumbai » pour la décennie à venir ouvre, ou non, de nouvelles perspectives de gouvernance métropolitaine.

\section{A l'image des métropoles indiennes, l'émergence d'une « ville-région »}

2 Malgré sa singularité, la trajectoire de Mumbai au cours des deux derniers siècles est exemplaire de l'émergence des métropoles indiennes.

\section{D'une ville insulaire ...}

3 C'est à partir du $17^{\mathrm{e}}$ siècle que Bombay devient une ville moderne avec un processus d'expansion rapide. Au cours du $19^{e}$ siècle, les changements sont spectaculaires : la population quadruple pour atteindre 928000 habitants en 1901 et la croissance économique s'accélère. Les grands ouvrages d'aménagement réalisés par le pouvoir colonial édifient des infrastructures de qualité permettant à Bombay de s'imposer comme la capitale économique de l'Inde au $20^{\mathrm{e}}$ siècle (Dossal, 1991). Des travaux de remblaiement et d'assèchement relient sept petits îlots entre eux qui deviennent la " island city ». Aujourd'hui cette " island city ", paradoxalement située tout au sud de la péninsule, reste le centre économique, historique et culturel de la métropole. Au cours du $20^{\mathrm{e}}$ siècle, le peuplement se poursuit sur la façade ouest tournée vers la mer, puis vers l'est le long du réseau ferroviaire. En 1961, ces banlieues sont incorporées dans la municipalité ${ }^{1}$. La ville de Mumbai est donc constituée de la "island city " (Mumbai district) et des banlieues (Mumbai suburban district) (voir carte 1). Selon le dernier recensement de 2001, Mumbai abrite 12 millions d'habitants et le déclin de la zone centrale, amorcé en 1961, se poursuit avec moins de 30\% de la population en 2001. 
Politiques publiques d'aménagement de la ville et de la métropole, croissance démographique naturelle et migrations contribuent à l'expansion en dehors de la municipalité. La ville nouvelle de Navi Mumbai, en projet depuis l'indépendance, a été aménagée par les autorités publiques. D'autres villes périphériques se construisent, souvent en bafouant les règles d'urbanisme. La métropole est un vaste espace composite, formé de territoires planifiés et aménagés, de territoires au développement anarchique et de zones interstitielles abritant bidonvilles, espaces semi-ruraux et zones d'emploi informel. Certaines villes de l'agglomération, comme Thane ou KalyanDombivili, ont elles-mêmes dépassé le million d'habitants et abritent en leur sein l'ensemble de cette diversité urbaine.

Schèma directeur de la région métropolitaine de Mumbai 1986-2011

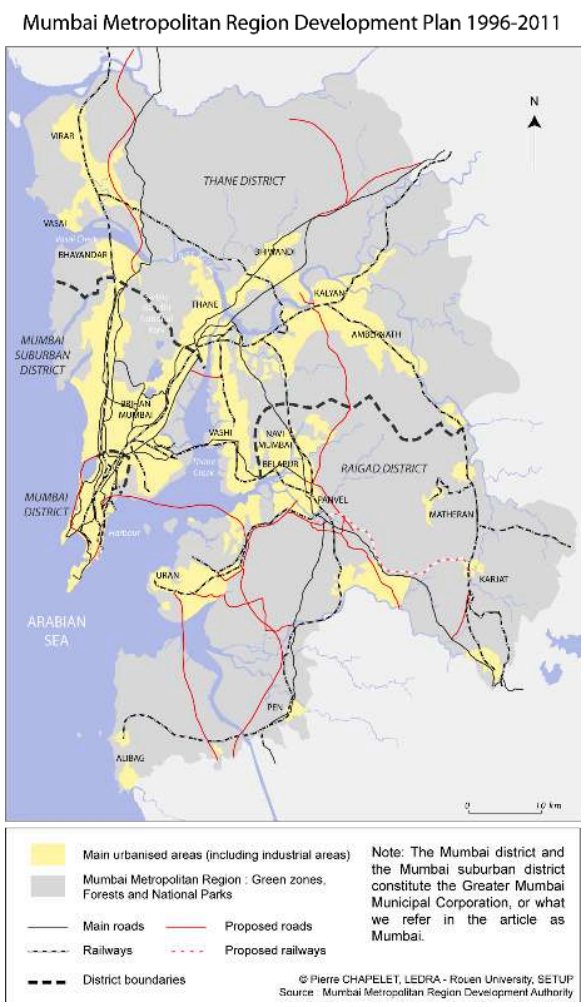

\section{... à une « ville-région » typique de la métropolisation en Inde}

Pour prendre en compte cette réalité de l'urbanisation hors de la «ville», le gouvernement crée en 1966 une agence métropolitaine d'aménagement, la Mumbai Metropolitan Regional Development Authority (MMRDA), responsable des schémas directeurs. La région métropolitaine est constituée de collectivités locales urbaines de différentes tailles dans les districts de Mumbai, Thane et Raigad. Elle comprend aussi de nombreux villages, situés dans la zone d'influence de l'urbanisation. Classiquement, la part de la population de Mumbai dans la région métropolitaine décline: le centre historique de la ville accueille aujourd'hui moins de $20 \%$ de la population de la région et la ville de Mumbai ne représente plus que deux tiers de la population de l'ensemble métropolitain. 
5 A ce stade, comment peut-on caractériser cet ensemble métropolitain, parmi les plus vastes au monde? Du point de vue économique, Mumbai est la métropole la plus riche de l'Inde. Elle représente à elle seule 4 à $5 \%$ du PNB. Les impôts perçus constituent $11 \%$ du budget total de l'Inde (Prud'homme, 2007). Le centre garde sa fonction de CBD et concentre les activités du monde de la finance et les sièges sociaux des grandes entreprises, malgré le déclin rapide de l'industrie textile. Ainsi, même si elle ne se situe pas dans le haut de la hiérarchie ${ }^{2}$, Mumbai est une ville globale. Néanmoins, si Mumbai prétend à ce statut, il nous semble qu'il ne permet pas de donner à voir sa spécificité. Comme les critiques de cette notion le soulignent (Robinson, 2006), les travaux sur la ville globale s'intéressent encore essentiellement aux seules dimensions économiques (Taylor, Catalano et al., 2002) ou à l'extrême concentration dans la zone centrale des villes de la pauvreté et des fonctions de contrôle et de commande (Sassen, 1994). Ce concept, dans le cas de Mumbai, éclipse les phénomènes de déconcentration des activités et des emplois vers les banlieues et les districts de Thane et Raigad, et les fortes inégalités socio-spatiales métropolitaines. Pour cette première raison, la notion de «ville région globale» de Scott semble a priori plus appropriée. La ville région est définie comme une zone métropolitaine centrale avec un hinterland de taille variable comprenant des territoires auxiliaires de développement moins dense et souvent caractérisés par des niveaux élevés d'inégalités de revenu et de bas salaires (Scott, 2001: 4-5). Dans la mesure où l'emprise spatiale urbaine de Mumbai dépasse largement la juridiction municipale qui n'est plus que le premier anneau d'un vaste ensemble urbain, la notion de "ville-région globale » est opératoire. Néanmoins, ce concept accorde une place centrale à la capacité de pilotage par un acteur politique métropolitain et à l'exercice d'une démocratie métropolitaine. Or, à Hyderabad, Kennedy et Ramachandraiah (2006) concluent que cet acteur politique métropolitain n'existe pas et en infèrent une spécificité des métropoles indiennes qui est un des obstacles à l'intégration métropolitaine. Ceci est-il le cas à Mumbai alors qu'y règne une forte compétition politique? En effet deux grandes coalitions s'y affrontent. La première est composée du Parti du Congrès et du Parti National du Congrès (émanation $\mathrm{du}$ Parti du Congrès après une scission en 1999). Ces deux partis dirigent ensemble l'Etat du Maharashtra depuis 2002. Ils ont donc le contrôle sur la définition des politiques urbaines et sur la MMRDA. Une deuxième coalition est constituée de la ShivSena (SS) (parti régionaliste et xénophobe hindou) et du Bharatiya Janata Party (BJP), le parti national de la droite hindoue. La Shiv Sena est très puissante dans les centres urbains du Maharashtra et gère plusieurs collectivités locales urbaines, dont celles de Mumbai et de Thane. In fine, la notion de "ville-région » permet de porter l'attention précisément sur les modalités institutionnelles et politiques de gestion de ces vastes ensembles urbains.

\section{Des lacunes dans la dimension « technique » du pilotage métropolitain}

6 On s'intéresse ici principalement à la dimension technique ou «fonctionnelle » du pilotage métropolitain, c'est-à-dire aux institutions qui produisent et gèrent les réseaux techniques, les programmes d'aménagement urbain et de logements. 


\section{Il n'y a pas de « vide institutionnel » mais une multiplicité d'organisations ...}

7 A l'échelle de Mumbai, la Corporation Municipale a été constituée dès 1888 suivant le modèle du "Commissioner System ». Le Commissaire Municipal dirige l'administration et les services municipaux. C'est un haut-fonctionnaire qui détient des pouvoirs supérieurs au conseil municipal. Le maire reste souvent dans l'anonymat. Il n'est qu'une figure symbolique élue par les conseillers municipaux. Cette structure bicéphale a permis au pouvoir colonial de gérer la ville tout en donnant un semblant de représentation aux notables locaux. Le fonctionnement actuel de la collectivité locale urbaine est un héritage postcolonial puisque c'est le gouvernement du Maharashtra qui nomme le Commissaire Municipal. La MCGM gère des dispensaires, des écoles municipales, le réseau de bus et d'électricité, l'eau et l'assainissement.

Avec le processus de métropolisation, d'autres municipalités gèrent leurs services urbains. Certaines prennent des initiatives sous l'impulsion de leur propre Commissaire Municipal. C'est le cas à Thane et à Navi Mumbai, villes relativement riches de l'espace métropolitain, qui ont lancé des programmes d'amélioration de la gestion de leurs services. En revanche, les collectivités locales urbaines plus petites ont une capacité de gouvernance moindre (manque d'expertise, finances locales faibles, services techniques réduits). D'une part, elles sont beaucoup plus dépendantes des transferts financiers de l'Etat pour leur fonctionnement. D'autre part, les réseaux techniques urbains qui requièrent de grands investissements sont conçus et financés par les organismes paraétatiques, en particulier par la MMRDA, qui peuvent alors leur imposer des conditionnalités sur les modes de gestion et de tarification des services urbains.

De facto, la Mumbai Metropolitan Regional Development Authority s'impose de plus en plus comme l'institution la plus importante à l'échelle métropolitaine. En sus de son rôle de planificateur, la MMRDA conçoit, planifie et met en œuvre de grands projets d'infrastructure. Dans la ville de Mumbai, elle devient un acteur central voire même incontournable puisqu'elle est l'agence coordinatrice des deux grands programmes d'amélioration des transports. Plusieurs facteurs expliquent le pouvoir de la MMRDA. D'abord, elle possède des terrains qu'elle revend aux enchères en lot pour des bureaux et des sièges sociaux. Ces transactions sur le marché de l'immobilier commercial, ajoutées à un fonds de roulement alimenté par l'Etat du Maharashtra, en font une agence métropolitaine riche, avec une forte capacité de financement. De plus, la Banque Mondiale, qui finance un des programmes de transport, a placé au centre du dispositif la MMRDA. Par ailleurs, la MMRDA, qui dépend du gouvernement du Maharashtra, s'avère être ainsi un instrument de contrôle pour celui-ci sur la région urbaine et sur Mumbai, qui est la plus riche des collectivités locales urbaines en Inde.

Il existe d'autres institutions avec des responsabilités plus sectorielles. C'est le cas de la MSRDC (Maharashtra State Road Development Corporation) établie comme entreprise publique en 1996 (ce qui permet des montages financiers spécifiques) pour construire l'autoroute entre Mumbai et Pune. Au fur et à mesure, ses compétences ont été élargies. Depuis plusieurs années, la MSRDC intervient à Mumbai en construisant des routes surélevées et deux grands ponts. Pour l'habitat, l'agence Maharashtra Housing and Development Authority construit des logements publics et le Slum Rehabilitation Authority s'occupe des questions de relogement et de réhabilitation. 
11 Lorrain (2007), dans son étude sur la métropole de Shanghaï, part de l'hypothèse que « le processus de construction des institutions s'appuie largement sur la nature des problèmes à résoudre ». Cette proposition peut s'appliquer à Mumbai où l'on peut lire la prolifération d'institutions comme une réponse à des problèmes spécifiques de différentes natures. Localement, on a créé une agence pour chaque problème. Mais cette dilatation institutionnelle s'inscrit aussi dans la logique nationale d'un Etat, acteur central du développement, qui a joué un rôle significatif en tant que pourvoyeur d'emplois.

\section{... évoluant dans un cadre fragmenté et compétitif}

12 Il n'y a donc pas de vide institutionnel à l'échelle métropolitaine. Caractéristique de toutes les grandes métropoles, la multiplicité des institutions est analysée en Inde comme un problème de fragmentation institutionnelle (Pinto, 2000). Pour valider ce postulat, la vraie question est de savoir comment ce foisonnement organisationnel fonctionne. En d'autres termes, est-il apte à anticiper et planifier les mutations métropolitaines ainsi qu'à définir les compétences et les frontières des institutions existantes?

Premièrement, il y a un problème de chevauchement et de manque de délimitations des responsabilités des institutions intervenant sur la zone métropolitaine. A cet égard, l'exemple du projet de pont autoroutier reliant le sud de Mumbai à Navi Mumbai est exemplaire (carte 2). Ce pont permettrait d'ouvrir l'hinterland vers le district de Raigad. Initialement, la MSRDC était responsable de la conception et du montage institutionnel de ce projet sous forme de BOT (Built-Operate-Transfer). Elle avait donc lancé un appel d'offres auquel plusieurs consortiums avaient répondu. Celui-ci, pour des raisons par ailleurs discutées (Leboucher, 2008), a totalement échoué si bien que le gouvernement du Maharashtra a décidé de financer lui-même ce projet à travers la MMRDA. Dans cette nouvelle répartition des rôles, la MSRDC reste en charge de la mise en œuvre, mais la MMRDA, qui a son propre département technique, cherche à élargir son emprise sur le projet au-delà de son simple rôle de financeur. Un tel projet devient alors peu lisible et rend opaque la responsabilité de ces différentes institutions. 


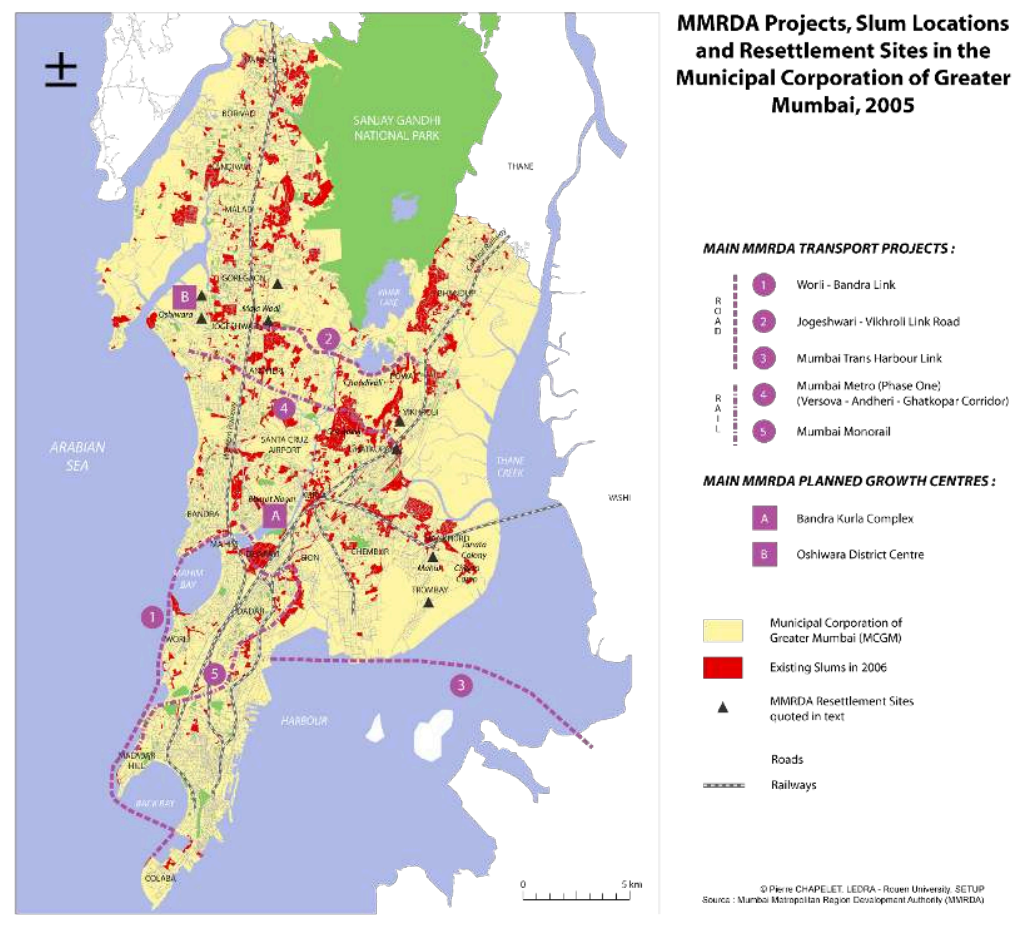

14 Deuxièmement, les mécanismes de coordination à l'échelle métropolitaine sont insuffisants. Ils se résument surtout au suivi de règles procédurales entre départements et administrations, et n'intègrent sous aucune forme les autres acteurs. Par conséquent, la majorité des projets urbains deviennent des sites de compétition entre institutions, qui est en particulier très forte entre la MMRDA et la MCGM. Les inondations de 2005 en sont un exemple criant $^{3}$. Les deux institutions se sont réciproquement reproché leur inaction en s'accusant l'une l'autre d'être responsables des délais dans les travaux publics d'assainissement et d'évacuation des eaux usées. Le diagnostic post-inondation a ensuite pointé du doigt le fait que la rivière Mithi n'est plus à même de jouer son rôle d'évacuation des pluies parce que son lit a été rétréci par les constructions (petites industries, bidonvilles, immeubles illégaux) et l'accumulation des ordures. Dans l'urgence, un projet d'élargissement de la rivière a été initié mais il se trouve pris dans des conflits d'intérêts exacerbés entre les deux institutions, et ce d'autant plus que la MMRDA a construit un nouveau quartier d'affaires sur une des rives de la rivière.

Enfin, alors que les institutions sont au cœur de ces dysfonctionnements structurels dans la conduite de l'action publique, elles sont par ailleurs confrontées à des revendications des acteurs de la société civile sous forme de pétitions, litiges en justice, parce qu'il n'existe pas de mécanismes d'intégration des demandes sociétales ou environnementales (Leboucher, 2008).

16 Le cadre d'action collectif à l'échelle métropolitaine est donc doublement défaillant. L'incapacité à discuter et à construire des consensus a minima, même entre institutions relevant in fine de l'Etat du Maharashtra, peut-il s'expliquer en partie par la complexité du pilotage politique de la métropole? 


\section{Qui gouverne la « ville-région » de Mumbai ?}

\section{La non application de la loi de décentralisation à l'échelle métropolitaine}

17 La mise en œuvre du volet urbain de la loi de décentralisation a permis de réelles avancées sur le respect des procédures électorales et la démocratisation du personnel politique. Néanmoins, on peut parler d'un inachèvement de la décentralisation en substance et en pratique, qui limite la construction d'un pilotage politique à l'échelle métropolitaine.

18 D'une part, le $74^{\mathrm{e}}$ amendement a prévu pour les métropoles la création d'un «Metropolitan Planning Committee ». Ce conseil métropolitain devrait être constitué aux deux tiers de membres élus des municipalités et inclure les élus des conseils villageois des zones rurales de l'agglomération. Il a pour tâche la coordination de la planification et l'aménagement de l'ensemble de la zone métropolitaine. Il s'agit donc en principe de transférer du pouvoir aux élus politiques et d'instituer les prémisses d'un gouvernement métropolitain. Mais à l'exception de Calcutta, aucune grande ville n'a appliqué ce volet de la loi pour le moment. En ce qui concerne Mumbai, la constitution de ce conseil métropolitain est en pourparlers depuis plusieurs années mais toujours repoussée, ce qui met en évidence la résistance du gouvernement du Maharashtra qui ne veut pas perdre une partie de son contrôle.

D'autre part, malgré la décentralisation, la capacité des élus locaux à piloter et à prendre en charge les fonctions de développement local est limitée par la bureaucratie et les échelons politiques supérieurs (Baud et De Wit, 2009; Ruet et Tawa Lama-Rewal, 2009). Par conséquent, même si les autorités locales sont représentées dans les " agences métropolitaines », elles sont dépourvues de pouvoir à cette échelle. Ainsi, le comité directeur de la MMRDA est composé essentiellement de membres des ministères du gouvernement. Cinq élus locaux et le Commissaire Municipal représentent Mumbai, alors que les élus des municipalités environnantes sont simplement invités à participer. Leur pouvoir est donc soumis à un échelon de décision supérieure qui est bien le gouvernement de l'Etat du Maharashtra, représenté par les élus de l'assemblée régionale.

\section{L'inachèvement de la décentralisation à l'échelle locale}

La loi de 1992 souhaitait renforcer la proximité entre élus et électeurs en introduisant à l'échelle infra-locale le principe de " comités de quartier » (les "ward committees »). A Mumbai, leur mise en place a été retardée jusqu'en 2000. Il n'en existe encore aujourd'hui que 16, pour 24 circonscriptions administratives. Chaque comité de quartier représente une population d'environ 240000 personnes, ce qui n'autorise pas l'émergence d'une forme de démocratie participative de proximité. De plus, le processus participatif est mis à mal car les ONG retenues pour participer à ces comités sont des organisations proches des responsables politiques locaux. Enfin, les pouvoirs donnés aux membres des comités et la portée de leurs décisions sont très limités par le type de projets et les montants qu'ils peuvent engager.

21 Pour remédier à cette situation, l'Etat indien a émis des directives pour l'application d'une « loi sur la participation communautaire » dans le cadre de la JNNURM. C'est aux 
Etats fédérés de mettre en œuvre cette législation. Cette proposition est très débattue au Maharastra car elle est défendue énergiquement à Mumbai par des associations de résidants puissantes qui ont un discours de plus en plus politisé. Bien que leur pouvoir effectif reste cantonné à certains arrondissements, leur capacité à se faire entendre sur leur conception idéale du Conseil métropolitain et des comités de quartiers est réelle (Zérah, 2007). Dans les deux cas, leur proposition est d'augmenter le nombre des représentants de la société civile. Cette suggestion est peu appréciée par les élus locaux qui souhaiteraient garder la mainmise. En effet c'est à cette échelle qu'ils bâtissent des réseaux d'influence et de patronage de redistribution des ressources.

La question du pilotage politique métropolitain est surdéterminée par le manque d'articulation (entretenu par l'Etat?) entre les échelles de gouvernance. A l'instar de toute métropole, Mumbai est une ville d'une extrême diversité. Aussi, chaque quartier ou localité a ses propres enjeux socio-économiques et ses propres modalités de gouvernance très ancrés localement. D'une part, ils ne sont pas reliés à une échelle supérieure, celle du conseil municipal et de l'échelle métropolitaine, puisque les élus locaux y sont dominés soit par les bureaucrates, soit par les élus de l'assemblée régionale. L'absence d'un réel espace de concertation métropolitain (forum ou conférence métropolitaine) ne permet pas d'impliquer ces acteurs "localisés » dans une logique de débat et de discussion sur les enjeux métropolitains, et encore moins les usagers qui sont marginalisés. D'autre part, entre l'échelle municipale et l'échelle de l'Etat, en l'absence d'une structure métropolitaine légitime politiquement, aucune innovation institutionnelle de collaboration entre municipalités (syndicats intercommunaux, conférence des maires) n’a émergé. Par conséquent la diversité urbaine, et encore plus celle des zones péri-urbaines, est mal prise en compte et on constate une absence d'emboîtement entre les niveaux de représentation politique. Ces désarticulations sont à comprendre dans le contexte politique très compétitif de la ville, puisque la coalition dominante à l'échelle du Maharashtra utilise les autorités métropolitaines pour définir les grands projets pour la ville et contrer la présence de la Shiv Sena localement. On peut en déduire que l'Etat ne souhaite pas dans ce contexte établir un conseil métropolitain mais préfère garder le contrôle à l'aide des institutions paraétatiques. Cette explication n'est toutefois pas suffisante et il nous faut avancer d'autres hypothèses explicatives. Il existe tout d'abord certainement une réelle défiance vis-à-vis du processus de décentralisation, visible dans l'organisation très hiérarchique des partis politiques. Par ailleurs, traditionnellement, la conquête du pouvoir politique ne se joue pas sur le terrain de la mise en œuvre de politiques publiques urbaines. Ce sont les enjeux identitaires et les questions développement régional (et avant tout rural) qui déterminent les règles de la compétition électorale. Le personnel politique ne s'investit donc que peu dans les enjeux urbains, la ville étant avant tout perçue comme une source de revenus. La question que pose la mise en avant d'une "Vision Mumbai » pour la ville est de déterminer si elle constitue un signe de rupture dans le traitement de l'ensemble métropolitain.

\section{La « Vision Mumbai » permet-elle de repenser l'échelle métropolitaine?}

23 Prise dans la compétition mondiale entre villes et voyant la montée en puissance de Delhi et des villes du sud du pays (Bangalore, Hyderabad, Chennai), le gouvernement du Maharashtra a initié au début des années 2000 un ambitieux projet de transformation 
de la ville : Vision Mumbai. Cette Vision a pour objectif de redonner de la compétitivité à une métropole qui a connu une décroissance économique à la fin des années 1990 . Comme nombre de ces ambitions réformatrices, en particulier pour les métropoles asiatiques, le programme promeut un modèle de coalition de croissance fondée sur de grands programmes d'infrastructure, des mesures de dérégulation foncière et l'augmentation des terrains constructibles. Un cercle vertueux se mettrait alors en place qui résoudrait les problèmes de logement et d'emploi, soutiendrait la mutation économique de Mumbai et réduirait mécaniquement la pauvreté. Cette vision a été très critiquée pour son approche élitiste qui valorise les espaces centraux de la métropole (Banerjee-Guha, 2009). Nous nous intéressons ici à la manière dont cette Vision pense l'échelle métropolitaine. Est-elle un instrument de remodelage du contenu métropolitain?

\section{Un constat classique : diagnostic a-spatial et des projets « intra muros » ...}

24 La «Vision Mumbai » fait tout d'abord un diagnostic des contraintes à l'attractivité de la métropole: lacunes en matière de planification urbaine et des politiques d'aménagement; déficit en services urbains et faible qualité de vie; manque d'intégration du marché du travail freinée par une mobilité réduite; manque de logements. Elle souligne par ailleurs fortement les problèmes de gouvernance et de sous-investissement chronique dans les infrastructures urbaines. En ce sens, on peut y voir une prise de conscience de la réalité métropolitaine. Pourtant, au vu de la transformation de la ville dans les dernières décennies, ce diagnostic n'est pas innovant. De plus, si l'on se penche sur l'analyse concrète de la métropole résultant des expertises et réunions de mise en œuvre, on constate que le document de «Vision Mumbai » dans sa version officielle (GOM, 2004) s'intéresse peu à la spécificité des différents territoires qui constituent la métropole. Mumbai n'y est d'ailleurs jamais définie de manière précise. Certes, la valorisation des espaces centraux, comme la transformation du bidonville de Dharavi ou des terrains des anciennes manufactures textiles, est au cœur d'un processus de gentrification (Banerjee-Guha, 2009). Toutefois, les propositions concernant la promotion de certains activités économiques ne discutent ni de leur localisation ni de leur capacité à créer des emplois pour réduire les inégalités territoriales métropolitaines. D'une certaine manière la prise de conscience de la dimension métropolitaine de Mumbai est posée de manière a-spatiale en partie par manque de production des connaissances. C'est seulement à partir de 2003-2004, lorsque la Banque Mondiale intervient comme conseiller auprès du gouvernement, qu'apparaissent les premières analyses économiques sérieuses (Prud'homme, 2005) et le financement d'un «business plan» régional (Mumbai Metropolitan Region Development Authority et Lea International Ltd, 2007).

Comme dans beaucoup de grandes villes, la question des transports, question métropolitaine par excellence, domine. En ce qui concerne les projets concrets prévus par ce plan, seuls deux parmi les plus importants se situent à l'échelle régionale : le premier est celui du nouvel aéroport dans le district de Raigad ; le second est le grand projet de pont sur la mer d'Arabie, reliant le sud de Mumbai à Navi Mumbai. Hormis ces exceptions, les gros investissements dans les transports urbains sont concentrés dans Mumbai «intra muros» $\left(\operatorname{carte}^{\circ} 2\right)$ : les projets de trois lignes de métro, le monorail, 
l'amélioration du réseau de routes et de la flotte de bus. L'amélioration du réseau ferroviaire concerne les banlieues mais les collectivités locales sont peu concernées et rarement consultées. Pour assurer la coordination entre l'ensemble des moyens de transport disponibles et faciliter l'intermodalité, une autorité métropolitaine des transports est officiellement proposée (United Metropolitain Transport Authority). Toutefois, elle n'a pas encore vu le jour et l'essentiel des efforts porte sur la dimension technique des projets.

Que centralité et constat d'ensemble (ou alors synthétique / général) soient mis en avant dans une «vision » qui a pour objet premier de mobiliser les acteurs de la ville n'est pas en soi étonnant. Il s'agit avant tout de définir une feuille de route. Néanmoins, à nos yeux, la vision stratégique contourne la question de la coordination entre les différents organismes et ne s'intéresse qu'à la marge à la résolution du problème des emboîtements d'échelle, comme le démontre l'immobilisme sur la gouvernance des transports. Pour comprendre l'absence d'analyse minutieuse et rigoureuse, il faut nous tourner vers le processus de formation et de mise en œuvre de Vision Mumbai.

\section{... Et un processus inapproprié : la cooptation plutôt que la consultation}

Au milieu des années 1990, le groupe «Bombay First » qui est composé des élites des grandes entreprises, à l'image de son équivalent "London First ", crée un cercle de réflexion sur la ville. En 2003, Bombay First commande une étude au cabinet de conseil McKinsey (Bombay First et McKinsey, 2003). Ce rapport est repris, ligne pour ligne, par le Gouvernement du Maharashtra pour Vision Mumbai, qui en adopte donc les conclusions. En 2005, la Banque Mondiale apporte son soutien et finance la création d'une cellule de travail pour la mettre en œuvre, le Mumbai Transformation Unit. Son rôle est de servir de soutien aux différents projets et de produire des recherches et des données sur la ville. Or la production d'expertise sur la ville est quasiment nulle. La faiblesse des analyses sur l'économie des villes n'est certes pas spécifique à Mumbai, néanmoins, à l'exception de commandes passées à des consultants internationaux chèrement payés et prompts à reproduire des «visions " inspirées d'autres villes, aucun mécanisme n'est mis en place pour s'appuyer sur les réseaux académiques et d'expertise locaux. De même, aucune réflexion n'est menée sur la possible refonte de l'architecture institutionnelle de l'action publique. Il n'y a pas de discussion sur la redéfinition des responsabilités ou la mise en place de mécanismes de coordination. Au contraire, il semble que chaque institution défende ses prérogatives plutôt que de contribuer à la mise en œuvre du projet de transformation de la ville.

Cette Vision est un instrument pour la coalition Congrès-NCP au pouvoir, afin de produire un discours fort sur la ville de Mumbai et d'éclipser la municipalité. Par ailleurs, elle a bénéficié du soutien du précédent chef du gouvernement du Maharashtra ${ }^{4}$, qui était très concerné par ses enjeux et conscient du poids croissant des circonscriptions électorales urbaines. Le gouvernement l'utilise avant tout comme un instrument de contrôle plutôt que comme un outil de mobilisation des acteurs, ce que montrent les deux structures de suivi qu'il a mises en place. La première, le Citizen Action Group, composé de quelques artistes et représentants de la société civile qui ont été cooptés, n'est en réalité qu'un semblant de processus participatif, Il ne fait que conforter le très fort déficit démocratique. Le deuxième mécanisme de suivi est un 
Empowered Comittee constitué de 17 représentants du gouvernement et de 8 représentants des grandes entreprises de Mumbai, qui utilisent cette opportunité pour promouvoir leur vision de la ville. Les représentants des petites industries, les syndicats ou les organisations professionnelles sont tous exclus de ce processus, qui relève d'une gouvernance par cooptation. Enfin, la Vision Mumbai est aussi pour l'Etat $\mathrm{du}$ Maharashtra un instrument de mobilisation des financements de la JNNURM pour de nombreux grands projets d'infrastructure. L'empilement des grands projets s'inscrit dans un mode d'action publique sclérosé, menée sans concertation et sans réflexion sur la refonte de l'architecture institutionnelle. A ce stade, on peut conclure que la «Vision Mumbai » ne contribue pas à (re)penser la gouvernance métropolitaine.

\section{Conclusion}

Le regard rapide que nous avons porté sur Mumbai souligne des constantes relatives à la question de la métropolisation en Inde. Tout d'abord, la prolifération d'institutions aux mandats qui se chevauchent et avec des légitimités contradictoires conduit à une fragmentation exacerbée par les enjeux politiques et un climat de compétition entre les institutions. La Vision Mumbai ne propose pas de pistes de réflexion pour une refonte de l'action publique qui s'adapte aux nouveaux enjeux. Elle vise avant tout à mobiliser de larges financements et à consolider le pouvoir de l'Etat du Maharashtra. Par conséquent, le processus de construction d'un acteur collectif métropolitain ne s'amorce pas. Le déficit démocratique métropolitain est évident dans l'absence de concertation et de forums de discussion. Il n'y a pas de régulation de la pluralité des acteurs et des intérêts. Au contraire, cooptation, lobbying et influence de certains groupes à différentes échelles caractérisent une gouvernance métropolitaine dans laquelle la nature des problèmes à résoudre ne fait pas l'objet d'un consensus suffisant pour permettre de repenser le rôle des institutions. Comme l'écrit alors dans le Business Standard K.C. Sivaramakrishnan ${ }^{5}$, le haut fonctionnaire responsable à la fin des années 1980 de la loi de décentralisation, le modèle actuel de fonctionnement des métropoles est insatisfaisant et «le modèle hiérarchique existant n'est ni sacré ni éternel ». Le défi des métropoles indiennes est bien d'ouvrir la boîte à idées pour trouver des outils de pilotage métropolitain adaptés à la réalité, tout en en renforçant la dimension démocratique.

\section{BIBLIOGRAPHIE}

Bagnasco A. et Le Galès P., 1997. Villes en Europe. Paris, Edition la DÂecouverte, 1 vol. 280p.

Banerjee-Guha S., 2009. Neoliberalising the 'Urban': New Geographies of Power and Injustice in Indian Cities. Economic and Political Weekly, XLIV, 22, p. 95-107.

Baud I. S. A. et J. De Wit, 2009. New forms of urban governance in India: Shifts, Models, Networks and Contestations. Delhi, Sage, 402p. 
Bombay First et McKinsey, 2003. Vison Mumbai. Transforming Mumbai into a world-class city. Mumbai, Bombay First: 32 .

Brenner N., 2004. New State Spaces. Urban Governance and the Rescaling of Statehood. Oxford University Press..

Dossal M., 1991. Imperial Designs and Indian Realities : The Planning of Bombay City, 1845-1875. Bombay, Oxford University Press, 251 p.

Dupont V., 2007. Conflicting stakes and governance in the peripheries of large Indian metropolises - An introduction. Cities, 24, 2, p. 89-94.

GOM, 2004. Transforming Mumbai into a World-Class City. First report of the Chief Minister's Task Force. Mumbai, Government of Maharashtra: 24.

Jouve B., 2005. La démocratie en métropoles: gouvernance, participation et citoyenneté. Revue Française de Sciences Politiques, 55, 2, p. 317-337.

Jouve B. et Lefèvre C., Eds.,2002. Métropoles Ingouvernables. Sociologie Economie Politique. Paris, Elsevier.

Kennedy L. et Ramachandraiah C., 2006. Logiques spatiales d'une stratégie régionale « high-tech ». L'exemple de HITEC City à Hyderabad (Inde) Flux, , 63/64, Innovations et territoires, p.54-70.

Kennedy L. et Zérah M.-H., 2008. The Shift to City-Centric Growth Strategies in India. Perspectives from Hyderabad and Mumbai. Economic and Political Weekly, XLIII, 39, p. 110-117.

Le Galès P., 2003. Le retour des villes européennes. Sociétés urbaines, mondialisation, gouvernement et gouvernance. Paris, Presses de Sciences Po, $454 \mathrm{p}$.

Le Galès P., 2005. Du gouvernement des villes à la gouvernance urbaine. Revue Française de Sciences Politiques, 45, 1, p. 57-95.

Leboucher P., 2008. Infrastructures à Mumbai. Etude de quatre projets d'aménagement: Bandra Worli Sea Link, Mumbai Trans Harbour Link, Mithi River Upkeep, Brimstowad. Delhi, ENTPECentre de Sciences Humaines: 43.

Lorrain D., 2007. Gouverner Shanghai (Une modernisation publique). Actors, Policies and Urban Governance. University of Paris X (Nanterre).

Mumbai Metropolitan Region Development Authority et Lea International Ltd, 2007. Business Plan for Mumbai Metropolitan Region. Draft Final Report. Mumbai, MMRDA.

Pinto M., 2000. Metropolitan City Governance in India. Delhi, Sage, 248 p.

Prud'homme R., 2005. Financing Mumbai Investment Needs. Note for the World Bank and the Mumbai Transformation Project: 15.

Prud'homme R., 2007. Seven Notes on Mumbai's Growth and How to Finance It. Unpublished: 66 Robinson J., 2006. Ordinary cities : between modernity and development. London ; New York, Routledge, xiv, $204 \mathrm{p}$.

Ruet J. et Tawa Lama-Rewal S., Eds.,2009. Governing India's Metropolises: Case Studies of Four Cities. Delhi, Routledge.

Sassen S., 1994. Cities in a World Economy. Thousand Oaks, California, Pine Forge Press, 157 p.

Scott A. J., Ed.,2001. Global City-Regions. Trends, Policy, Theory. Oxford, University Press

Taylor P. J., Catalano G. et Walker D. R. F., 2002. Measurement of the World City Network. Urban Stud, 39, 13, p. 2367-2376. 
Zérah M.-H., 2007. Middle Class Neighborhood Associations as Political Players in Mumbai. Economic and Political Weekly, XLII, 47, p. 61-68.

\section{NOTES}

1. Dans ce texte, on l'appellera indifféremment la ville de Mumbai ou MCGM pour Municipal Corporation of Greater Mumbai.

2. Le classement des villes réalisé par le Globalization and World Cities Research Network (http://www.lboro.ac.uk/gawc/index.html) comprend plusieurs catégories et trois rangs pour les villes globales. Entre 2004 et 2008, Mumbai est passée du rang 4 au rang 3. Les villes de rang 3 sont définies comme des villes mondiales de grande importance qui relient des régions économiques entre elles et favorisent l'intégration des économies nationales dans l'économie mondiale.

3. Le 26 juillet 2005, une pluie de mousson centennale conjuguée à la marée haute ont provoqué des inondations qui ont fait plus de 1000 morts. Un concert de voix unanime s'est élevé fustigeant l'apathie des autorités publiques.

4. Il a perdu son poste à la suite des attentats du 26 novembre 2008.

5. K C Sivaramakrishnan: Megacity Governance disponible sur http://www.businessstandard.com/india/news/k-c-sivaramakrishnan-megacity-governance/351164/

\section{RÉSUMÉS}

In India, economic liberalization and decentralization create the conditions for the rise of cities. As in other parts of the world, the metropolization process is accelerating. It raises the question of the formation of cities as a collective actor. The empirical analysis is based on the case of Mumbai. Pushed by international competition, the policy makers have launched a major reform program: Vision Mumbai. First, the article examines the issues and content of metropolitan governance. On the other hand, it seeks to assess the ability of such a vision to open new avenues for reflection on the future of metropolitan governance and mobilize all stakeholders in the city.

\section{INDEX}

Mots-clés : Asie du Sud, Inde, urbanisme, gouvernance métropolitaine, Mumbai

Keywords : South Asia, India, urban studies, metropolitan governance, Mumbai

\section{AUTEUR}

\section{MARIE-HÉLÈNE ZÉRAH}

Marie-Hélène Zérah, (zerah@ird.fr) est chargée de recherche à l'IRD, détachée actuellement au Centre de Sciences Humaines de New Delhi. Elle a publié récemment :

- Zérah M.-H., 2009. Reforming solid waste management in Mumbai and Hyderabad: Policy 
convergence, distinctive processes. in J. Ruet et S. Tawa Lama-Rewal, Governing India's Metropolises: Four case studies. Delhi, Routledge: p. 241-269.

- Zérah M.-H., 2009. Politique et gouvernance dans le secteur de l'eau en Inde : le cas de Mumbai. in G. Schneier-Madanes, L'eau mondialisée: la gouvernance en question. Paris, La Découverte (à paraître)

- Zérah M.-H., 2008. Splintering urbanism in Mumbai: Contrasting trends in a multilayered society. Geoforum, 39, 6, p. 1922-1932.

- Kennedy L. et Zérah M.-H., 2008. The Shift to City-Centric Growth Strategies in India. Perspectives from Hyderabad and Mumbai. Economic and Political Weekly, XLIII, 39, p. 110-117. En Inde, la libéralisation économique et la décentralisation créent les conditions de la montée en puissance des villes. Comme pour d'autres régions du monde, le processus de métropolisation s'accélère et pose la question de la constitution des villes comme acteur collectif. L'analyse empirique s'appuie sur le cas de Mumbai, qui, entraînée dans la compétition internationale, a lancé un grand programme réformateur : Vision Mumbai. D'une part, l'article examine les enjeux et le contenu de la gouvernance métropolitaine. D'autre part, il cherche à évaluer la capacité d'une telle vision à ouvrir de nouvelles pistes de réflexion sur le futur de la gouvernance métropolitaine et à mobiliser tous les acteurs de la ville. 